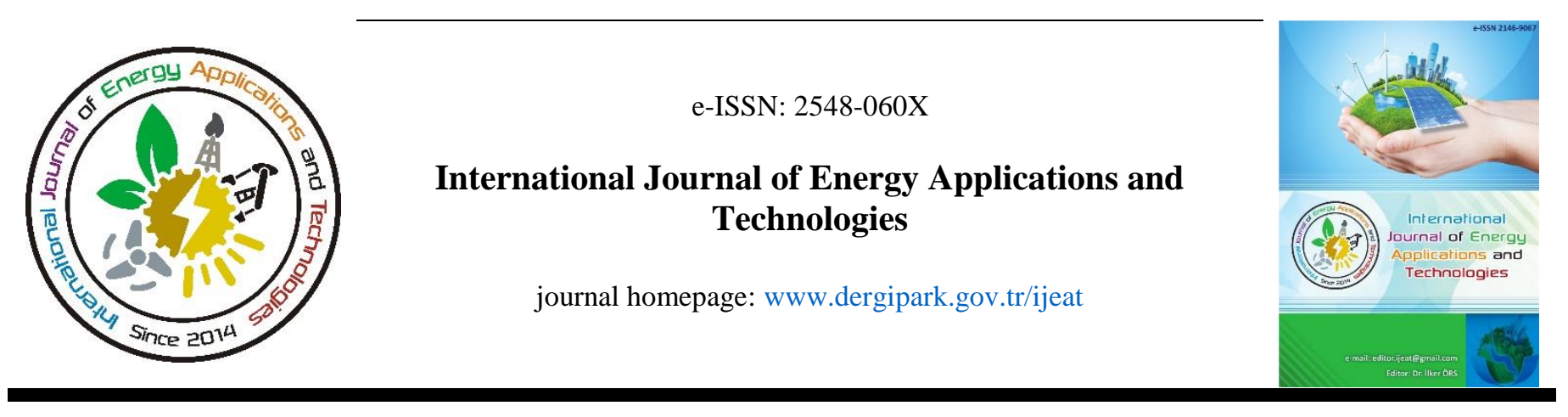

Original Research Article

\title{
Robust uncertainty modelling of energy variations in electric motors for efficient and reliable mechanical structural analysis
}

\author{
Murat Mayda \\ Mechanical Engineering Department, Karamanoglu Mehmetbey University, Faculty of Engineering, TURKEY
}

ARTICLE INFO
* Corresponding author
mmayda@ kmu.edu.tr
Received March 1, 2018
Accepted March 28, 2018
Published by Editorial Board
Members of IJEAT
C) This article is distributed by
Turk Journal Park System under
the CC 4.0 terms and conditions.

\begin{abstract}
The mechanical power and rotational speed of electric motors have significantly uncertainties arising from energy variations such as the magnetic energy, the flux linkage variation of the winding, and the air gap due to the rotor position. The main aim of this work is to address the effect of the uncertain output power and rotational speed of an electric motor on mechanical structural analysis, especially on the torsional analysis, and accordingly to model the statistical characteristics of variations in torque of the motors in consideration of different powers and speeds for further efficient and reliable mechanical structural analysis under uncertainty. To perform these tasks, a case study that is the torsional loading of a shaft by an electric motor and generator, is carried out. The results show that the uncertainty of power and speed in electric motors considerably affects the probability of failure of the shaft in case of exceeding the maximum shear stress, and increasing the speed at a given power does not significantly change the $\mathrm{COV}$ value of the torque whereas increasing the power at a given speed can relatively change the COV value of the torque. The obtained average of the COV values $(0.0023)$ of torque with normal distribution is fairly sufficient for indicating the variations in the torque of electric motors. Moreover, the obtained torque uncertainty can be easily and efficiently used in the mechanical structural analysis under both deterministic and stochastic cases.
\end{abstract}

Keywords: Electric motors, energy variations, uncertainty, modelling, structural analysis

\section{Introduction}

An electric motor is an electromechanical device that converts electric energy into mechanical energy, and produces mechanical power and rotational speed to mechanical systems. Most of the conducted research on electric motor have concentrated on the motor energy efficiency optimization [1-4]. However, the generated powers and speeds by electric motors have significantly uncertainties arising from energy variations such as the magnetic energy, the flux linkage variation of the winding, and the air gap due to the rotor position [5-7]. Therefore, taking into consideration of these uncertainties and errors in the motor efficiency estimation and measurement has emerged as an important necessity for the development of the motors, and the mechanical systems associated with them [8]. For that purpose, Aarniovuori et al. [9] investigated the uncertainties in both electric and mechanical power, and in speed data belonging to five different sizes of inductive electric motors. Similarly, $\mathrm{Li}$ et al. [10] examined the uncertainties in the electric power and rotational speed data belonging to four different sizes of inductive electric motors. Even though these works have made significant contributions to the necessity of consideration of uncertainty in the factors of the motors, there is still needed to conduct a detailed stochastic analysis of the uncertainties of factors in the motors, and thus to model the stochastic characteristics. By this way, it can be possible to implement more reliable and 
efficient mechanical structural analysis associated with the motors under uncertainty [11-13]. In this work, how to address the effect of the uncertain output power and speed data of an inductive electric motor on mechanical structural analysis, especially on the torsional analysis, and to model the statistical characteristics of variations in torque of the motors in consideration of different powers and speeds for further efficient and reliable mechanical structural analysis under uncertainty are investigated by using MCS (Monte Carlo Simulation).

The rest of this work is organized as follow: in Section 2, the robust uncertainty modelling of output power and rotational speed of electric motors are realized. In Section 3, the mechanical structural analysis under the stochastically modelled variables of electric motors, and modelling the torque uncertainty via a case study is implemented. In Section 4, a conclusion explaining the contributions of this work is briefly drawn.

\section{Uncertainty Modelling of Output Power and Rotational Speed of Electric Motors}

In this section, a short explanation about the statistical information to model the uncertainty in a random variable was given, and the experimental data collected from the literature were processed in such a way that is able to represent the power and speed data as standard uncertainties. The Coefficient of Variation (COV), $\delta_{X}$, which is known to be a measure of uncertainty or the relative amount of uncertainty in a variable, and is given by the following formula:

$$
\delta_{X}=\frac{\sigma_{X}}{\mu_{X}}
$$

Where, $\sigma_{X}$ and $\mu_{X}$ are the standard deviation and mean of a random variable $X$, respectively.

At the result of the detail literature search, totally nine power data collected separately from the references [9] and [10], which are the mechanical or output power of the electric motors $\left(P_{\text {mech }}\right.$ in $\left.\mathrm{kW}\right)$ and the combined standard uncertainty with respect to $P_{\text {mech }}\left(u\left(P_{\text {mech }}\right)\right.$ in $\left.\mathrm{W}\right)$, were found. Afterwards, a $\mathrm{COV}$ value for each power was calculated by the ratio of $u\left(P_{\text {mech }}\right)$ to $P_{\text {mech }}$ (in $\left.\mathrm{W}\right)$. All of these data are presented in Table 1 . The mean and standard deviation of all COV values of the nine collected output power data were calculated to be 0.0014 and 0.00045 , respectively. For the confidence interval of $95 \%(\mathrm{k}=2)$ with normal distribution, the uncertainty of $\mathrm{COV}$ for power can be expressed as $0.0014 \square 0.0009$. Therefore, the maximum value of $\mathrm{COV}$ within the confidence interval of $95 \%$ was assumed to $0.0023 \mathrm{~W}$ $(0.0014+0.0009)$.

Li et al. [10] also experimentally measured the uncertainty in the rotational speed of $3000 \mathrm{rev} / \mathrm{min}$ as $u\left(n_{\text {mech }}\right)=0.0508$ $\mathrm{rev} / \mathrm{min}$. In this case, for the confidence interval of $95 \%(\mathrm{k}=2)$ with normal distribution, the COV for rotational speed can be expressed as $0.0000339(0.1016$ / 3000).

Table 1. The works conducted on power measurement uncertainty of inductive electric motors

\begin{tabular}{cccc}
\hline Work conducted & $\boldsymbol{P}_{\text {mech }}(\mathbf{k W})$ & $\boldsymbol{u}\left(\boldsymbol{P}_{\text {mech }}\right)(\mathbf{W})$ & $\mathbf{C O V}$ \\
\hline & 7.5 & 14 & 0.00187 \\
& 15 & 27 & 0.00180 \\
Aarniovuori et al. [9] & 37 & 71 & 0.00192 \\
& 90 & 142 & 0.00158 \\
& 200 & 289 & 0.00145 \\
\hline \multirow{4}{*}{ Li et al. [10] } & 7.5 & 11.2 & 0.00149 \\
& 14 & 11.2 & 0.00080 \\
& 55 & 77 & 0.00140 \\
& 315 & 204.75 & 0.00065 \\
\hline
\end{tabular}

Stochastic representations of the output power and rotational speed, which are the mean, standard deviation and distribution type of them, were presented in Table 2.

Table 2. Stochastic representation of the output power and rotational speed

\begin{tabular}{lccc}
\hline \multicolumn{1}{c}{ Parameter } & Mean & Standard deviation & Distribution \\
\hline$P_{\text {mech }}(\mathrm{W})$ & $\mu_{P \text { mech }}$ & $\mu_{P \text { mech }} \times 0.0023$ & Normal \\
$n_{\text {mech }}(\mathrm{rev} / \mathrm{min})$ & $\mu_{n \text { mech }}$ & $\mu_{n \text { mech }} 0.0000339$ & Normal \\
\hline
\end{tabular}

3. Mechanical Structural Analysis under the Stochastically Modelled Variables of Electric Motors

In this section, a case study was carried out to illustrate the effect of the uncertain output power and speed data of an electric motor on the torsional loading of a shaft, and also to model the statistical characteristics of variations in torque in consideration of different powers and speeds.

As an example, the hollow steel shaft has an outer diameter of $30 \mathrm{~mm}$, and it will transmit $45 \mathrm{~kW}$ of power from the engine to the generator when rotating at $1000 \mathrm{rev} / \mathrm{min}$ as can be seen in Figure 1. The problem herein is to determine the greatest inner radius of the shaft under deterministic and stochastic cases if the allowable shear stress is $\tau_{\text {allow }}=140$ MPa.

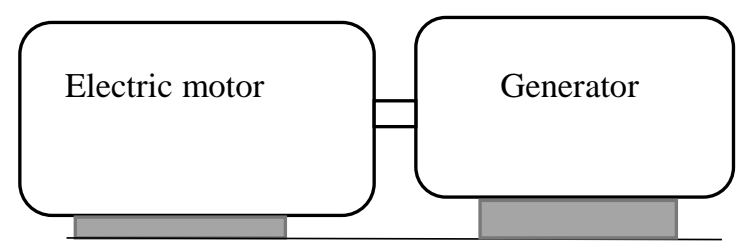

Figure 1. A hollow steel shaft with torsional load applied by electric motor and generator

As for the power transmission, the power $(P$ in $\mathrm{W})$ equals the product of torque $(T$ in $\mathrm{Nm})$ and speed ( $\omega$ in $\mathrm{rad} / \mathrm{s})$, as expressed below in Equation (1).

$$
P=T \omega
$$


As regard to torsional strength of a hollow shaft, the maximum shear stress $\left(\tau_{\max }\right)$ is calculated by

$\tau_{\max }=\frac{T c}{J}$

Where $J=\frac{\pi}{2}\left(r_{o}^{4}-r_{i}^{4}\right), J$ is the polar second moment of area, $r_{o}$ and $r_{i}$ is the radius of outer and inner diameter of the shaft, respectively. $c$ is the distance from the axis of the shaft, and equals to the $r_{o}$ for shafts.

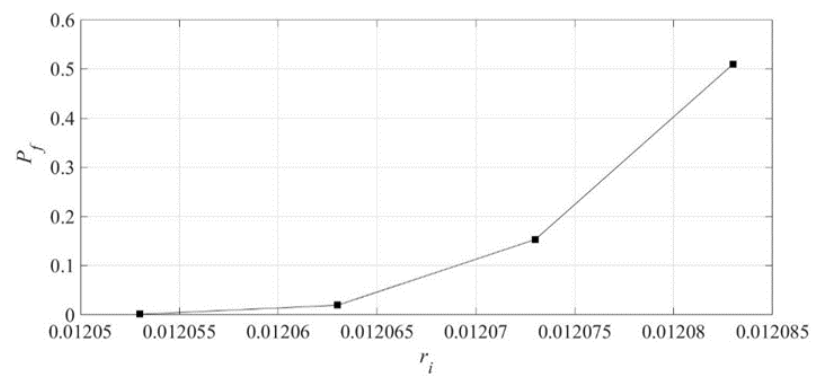

Figure 2. The $P_{f}$ values versus the $r_{i}$ values

Under determinist case, the ri value was found to be 0.012083 $\mathrm{m}$ from Equation (2) and (3). However, the ri of $0.012083 \mathrm{~m}$ leads for the shaft to a Pf of about $0.50(50 \%)$ in the shaft design under stochastic case using MCS with 1000000 realizations. To find more reliable ri values following the stochastic procedure with MCS, Pf values were calculated decreasing the ri value. The results of the Pf values versus the ri values are presented by a plot graph in Figure 2, and consequently one of the most promising ri value was found to be $0.012053 \mathrm{~m}$ with the Pf of $9 \mathrm{e}-04$ (referred to be $99 \%$ reliable). From the results, we conclude that the uncertainty of output power and rotational speed in electric motors considerably affects the mechanical structural analysis.

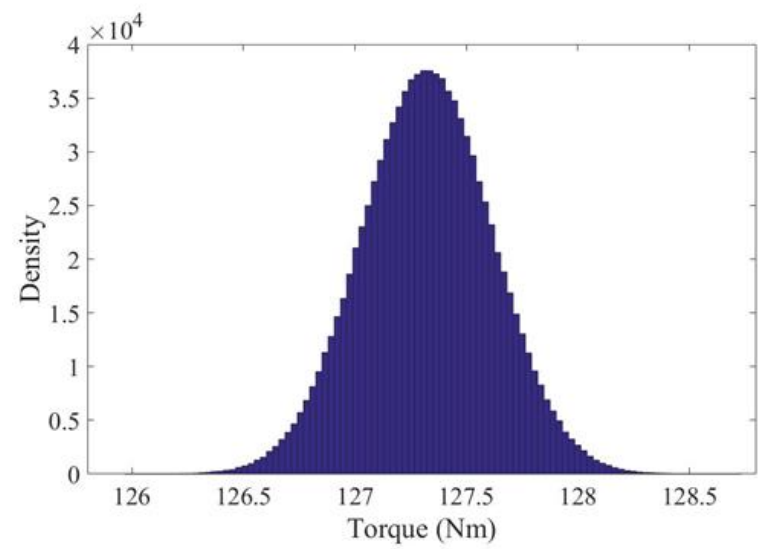

Figure 3. Normal distribution of the torque obtained at the given deterministic design point

Statistically modelling the uncertainty of output power and rotational speed in electric motors can contribute to an efficient mechanical structural analysis; otherwise, it is required to conduct the same computationally expensive simulations to obtain the statistical results, prior to reliable design under uncertainty. To that end, the statistical characteristics of variations in torque in consideration of different powers and speeds were examined. First, the distribution of the torque was obtained at the given deterministic design point, which is Normal distribution as can be seen in Figure 3.

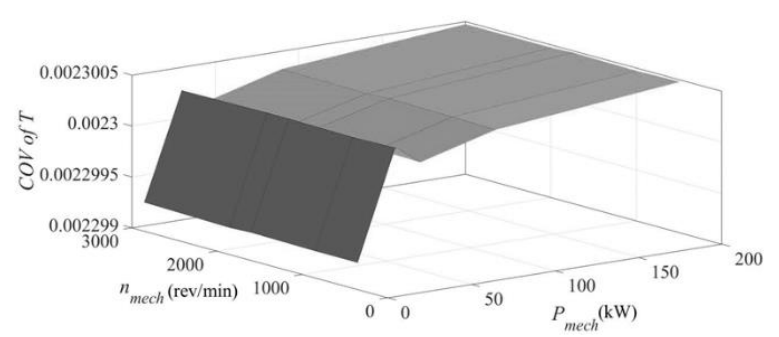

(a)

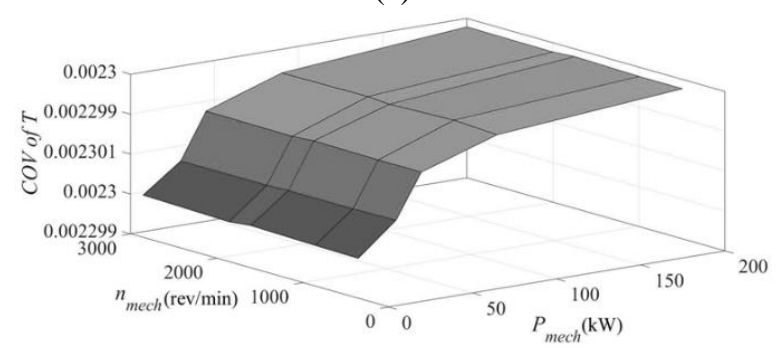

(b)

Figure 4. Two trials on COV of the torque against output power and speed ( $a$ and b) using MCS with 10000000 realizations

Second, with the aim of examining the COV variation of the torque against different output powers $(7.5,30,45,90,200$ $\mathrm{kW}$ ) and speeds (500, 1000, 1750, 2000 and $3000 \mathrm{rev} / \mathrm{min})$ under the uncertainty, a five-levels full factorial design, which includes 2 factors ( $P_{\text {mech }}$ and $\left.n_{\text {mech }}\right), \mathrm{L}=5$ levels per factor, and thus totally 25 samples, was implemented. Two trials on COV of the torque against output power and speed based on the data obtained from the experimental design by using MCS with 10000000 realizations are shown in Figure $4 \mathrm{a}$ and $4 \mathrm{~b}$. According to the 3D graphs, increasing the speed at a given power does not significantly change the COV value of the torque whereas increasing the power at a given speed can relatively change the COV value of the torque. However, the small changes in the COV value can be ignored because there is no significant changes (to 5 or 6 decimal places). As a result, the average of the COV values (0.0023) is fairly sufficient for indicating the variations in the torque of electric motors. Therefore, the torque uncertainty of a COV of 0.0023 stemming from the uncertainty in the output power and rotational speed of electric motors can be easily used in the mechanical structural analysis under both deterministic and stochastic cases. 


\section{Conclusion}

In this work, considerable attention was given to two main aspects: (1) addressing the effect of the uncertain output power and speed data of an inductive electric motor on mechanical structural analysis, especially on the torsional analysis, and (2) modelling the statistical characteristics of variations in torque of the motors in consideration of different powers and speeds for further efficient and reliable mechanical structural analysis under uncertainty if required. To address these two aspects, a case study that is the torsional loading of a shaft by an electric motor and generator, was carried out. The results simulated by MCS show that from the first aspect, we conclude that the uncertainty of output power and rotational speed in electric motors considerably affects the mechanical structural analysis. From the second aspect, increasing the speed at a given power does not significantly change the $\mathrm{COV}$ value of the torque whereas increasing the power at a given speed can relatively change the COV value of the torque. However, the small changes in the COV value can be ignored because there is no significant changes (to 5 or 6 decimal places); therefore, the average of the COV values $(0.0023)$ of torque with normal distribution is fairly sufficient for indicating the variations in the torque of electric motors. Thus, the obtained torque uncertainty of a COV of 0.0023 stemming from the uncertainty in the output power and rotational speed of electric motors can be easily and efficiently used in the mechanical structural analysis under both deterministic and stochastic cases.

\section{References}

[1] Farhani, F., Zaafouri, A. and Chaari, A., 2017, "Real time induction motor efficiency optimization", Journal of the Franklin Institute, 354(8): 3289-3304.

[2] Verucchi, C., Ruschetti, C., Giraldo, E., Bossio, G. and Bossio, J., 2017, "Efficiency optimization in small induction motors using magnetic slot wedges", Electric Power Systems Research, 152: 1-8.

[3] Hannan, M.A., Ali, J.A., Mohamed, A. and Hussain, A., 2018, "Optimization techniques to enhance the performance of induction motor drives: A review", Renewable and Sustainable Energy Reviews, 81: 1611-1626.

[4] Çelik, M., Örs, İ., Bayindirli, C. and Demiralp, M., 2017, "Experimental investigation of impact of addition of bioethanol in different biodiesels, on performance, combustion and emission characteristics", Journal of Mechanical Science and Technology, 31(11): 5581-5592.

[5] Kim, S.-H., "Chapter 4 - Modeling of alternating current motors and reference frame theory", in Electric Motor Control. 2017, Elsevier. p. 153-202.

[6] Yu, J., Zhang, T. and Qian, J., "5 - Measurement errors and uncertainties", in Electrical Motor Products. 2011, Woodhead Publishing. p. 81-172.
[7] Marino, R., Tomei, P. and Verrelli, C.M., 2008, "An adaptive tracking control from current measurements for induction motors with uncertain load torque and rotor resistance", Automatica, 44(10): 2593-2599.

[8] Lu, B., Cao, W. and Habetler, T.G. "Error Analysis of Motor-Efficiency Estimation and Measurement", in 2007 IEEE Power Electronics Specialists Conference. 2007.

[9] Aarniovuori, L., Kolehmainen, J., Kosonen, A., Niemelä, M. and Pyrhönen, J. "Uncertainty in motor efficiency measurements", in 2014 International Conference on Electrical Machines (ICEM). 2014.

[10] Li, F., Zhang, Y., Li, J., Yang, X., Li, T. and Shang, W. "Application of Measurement Uncertainty for Electric Motor Efficiency Evaluation", in International Forum on Energy, Environment Science and Materials. 2015.

[11] Choi, S.-K., Grandhi, R.V. and Canfield, R.A., 2007, "Reliability-based Structural Design: Springer-Verlag London.

[12] Mayda, M., 2017, "An Efficient Simulation-Based Search Method for Reliability-Based Robust Design Optimization of Mechanical Components", MECHANIKA, 23(05): 696-702.

[13] Mayda, M. and Choi, S.-K., 2017, "A reliability-based design framework for early stages of design process", Journal of the Brazilian Society of Mechanical Sciences and Engineering, 39(6): 2105-2120. 